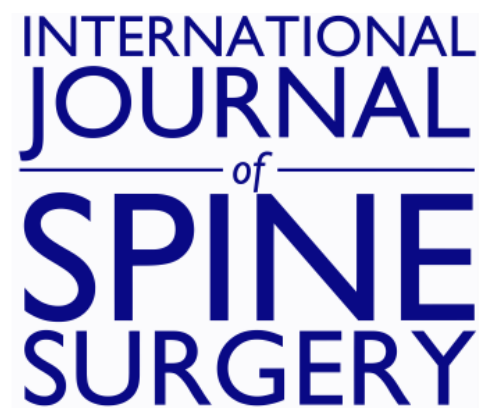

\title{
Clinical Symposium II: Interspinous-based Dynamic Stabilization
}

Dieter Adelt, Jean Taylor, James Zucherman and Neel Anand

Int J Spine Surg 2008, 2 (3) 150-154

doi: https://doi.org/10.1016/SASJ-2008-Symposium3

http://ijssurgery.com/content/2/3/150

This information is current as of April 26, 2023.

Email Alerts Receive free email-alerts when new articles cite this article. Sign up at:

http://ijssurgery.com/alerts

The International Journal of Spine Surgery

2397 Waterbury Circle, Suite 1,

Aurora, IL 60504, Phone: +1-630-375-1432

(C) 2008 ISASS. All Righfso Refservededom http://ijssurgery.com/ by guest on April 26, 2023 


\title{
Clinical Symposium II: Interspinous-based Dynamic Stabilization
}

\author{
Clinical Symposia Deputy Editors: Neel Anand, MD, and Harvinder Sandhu, MD
}

PANEL MEMBERS:

Dieter Adelt, MD
Ostseeklinik, Damp, Germany

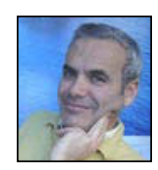

Jean Taylor, MD

Princess Grace Hospital of Monaco

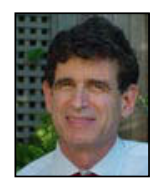

James Zucherman, MD

St. Mary's Spine Center, San Francisco, California

\section{INTRODUCTION}

\section{Neel Anand, $M D^{a}$}

Interspinous-based dynamic stabilization has become a popular form of treatment in recent years. Though many companies have developed innovative designs for interspinous spacer devices, there are many questions yet to be answered. Some of these devices have considerably more clinical experience than others, but their role in the treatment of spinal pathologies is still controversial.

The mechanism of action in itself has not been fully elucidated, though many theories have been proposed. Validation of these theories becomes important to determining the appropriate indications for use of these devices. Without a clear understanding of the mechanism of action or what these implants really do, their future would remain questionable. The general consensus seems to be that these devices help by unloading the facet joints and thereby have a role to play in treating back pain arising from facet arthrosis. Others have used a spacer device to indirectly decompress the spinal canal in spinal stenosis. The effect on the disc itself is still largely undetermined.

The stability provided by these devices is also unknown and has led to some innovative designs including tethers that bind the device to the spinous processes. Wings and flanges have been designed to keep the device in place and the importance of maintaining the interspinous ligament post implantation is indeed unknown.

Although only one spacer has been FDA-approved for use in the US, we now have several years of follow-up data on hundreds of patients from well-designed IDE studies of multiple devices. Other devices have received the CE mark in Europe. Changes have been made to designs and materials in several spacer devices.

To help us better understand the mechanisms and design challenges of some of these devices and to get a fresh update on the results of ongoing testing, we went to 3 esteemed surgeons who have been instrumental in the development, refinement, and testing of 3 of the interspinous spacers: Coflex, Diam, and X-Stop. Their insight into this technology is presented in this symposium for our readers.

Key Words: interspinous spacer; spinal stenosis; interspinous implant; dynamic stabilization; facet arthrosis. SAS Journal. Summer 2008;2:150-154. DOI: SASJ-2008-Symposium3

${ }^{a}$ Cedars Sinai Medical Center, Los Angeles, California

Panel members Drs. Adelt and Taylor are consultants to device manufacturers and/or have financial interest in the respective device. 


\section{Question \#1. Describe the interspinous spacer device(s) you have had experience in using. How have the devices evolved?}

Adelt: For $6 \frac{1}{2}$ years we have been working with the Coflex system (Paradigm Spine, LLC, New York, New York). We have used it for protecting adjacent levels in fusion and-and this is the main indication - for back pain in spinal stenosis. Over that time span, we have operated on more than 600 patients, and the device has evolved as an adjunctive of or as the treatment for low-back pain coming from arthrotic facet joints. This is a situation of spinal canal stenosis, so there is a close relationship between stenosis - low-back pain (LBP) and Coflex. The philosophy (current indication in the US FDA investigational device exemption trial) is to decompress the spinal canal and then implant the interlaminar/interspinous implant for stabilization after microsurgical decompression.
Taylor: The Diam System interspinous process distraction (IPD) device (Medtronic Sofamor Danek, Memphis, Tennessee) is a deformable damper made of silicone, with a polyester jacket. It is designed with oversized wings, which contribute to the stability and performance of the implant.
Zucherman: I have been using the X-Stop interspinous spacer (Kyphon Inc., subsidiary of Medtronic, Sunnyvale, California) since 1997. We have done approximately 500 cases. The first embodiment of the device had a rectangular loading element, which was soon designed to be elliptical and revolving. This all-titanium device has been used extensively in Europe and the US.

The X-Stop PK was designed approximately 5 years ago. It has been released and used in Europe for several years and is expected to be generally released in the US in the near future. The X-Stop PK device has a more sophisticated loadbearing element, which involves a polyetheretherketone (PEEK) circumferential ring around a titanium core. The
PEEK material, which more resembles bone in the modulus of elasticity, is also made at the appropriate thickness so that under loads, a small amount of deformation occurs, which increases surface contact as much as fourfold, thus diminishing the bone-loading characteristics. We felt this design would minimize some of the aspects of settling of the device into the spinous process or resorption, which we believe is a factor in the outcome, especially in osteopenic patients and very active patients. Additionally, the footprint of the loading element in the X-Stop PK is broader, also further diminishing load on the spinous process-bone implant interface.

I have not had the pleasure of utilizing any of the other interspinous devices.

\section{Question \#2. What are the challenges of designing an effective interspinous spacer?}

Adelt: The therapy for spinal canal stenosis is microsurgical decompression. The challenge for an interspinous spacer is for the implantation to be performed via a minimally invasive procedure, but this procedure depends on the decompression procedure. The implant must be a dynamic one. It is imaginable for an implant to not touch the supraspinous ligament. Due to anatomical findings and biomechanical reasons, we wish to have a tool to allow us to treat potentially unstable segments with interspinous spacers. Indications, although not proven, included LBP in stenosis, adjacent segment protection, recurrent herniated nucleus pulposus (HNP) and primary voluminous HNP.
Taylor: The first real challenge is finding a compromise between stiffness of the device and its interaction with the surrounding bony environment. Second, the device is intended to restore painless motion without affecting physiologic function. Lastly, the procedure has to be minimally invasive and cost-effective.
Zucherman: Interspinous spacers work by repositioning or containing motion of the spinous processes that depend on the strength of the connection between the spinous process and the middle and anterior spinal column for their effect. Therefore, the strength of the spinous process in its connection to the posterior elements poses a limit on what one can accomplish with the interspinous spacers. Increasing the strength of the interspinous-posterior element connection could increase the effectiveness of the interspinous spacer devices. In order to prevent excessive flexion of the treated segment, one must leave the supraspinatus ligament intact or place spinous process straps in position. The distance of these posterior tension bands to the loading element determines their cantilever and unloading effect on the middle and anterior spinal columns. If a strap is placed, then a flexion limitation is added to the implant, which may or may not be desirable, depending on the indications to be treated. 


\section{Question \#3. What do you believe is/are the mechanism(s) of action of these devices and is there evidence to support} the same?

Adelt: The device unloads the facet joints and maintains foraminal height, and the Coflex is very near to the center of rotation of the segment secondary to its interlaminar/ interspinous positioning. The range of motion is decreased, resulting in the improvement of low-back pain due to "controlled restricted motion." While other implants lay claim to direct therapy of canal stenosis, all are not dynamic. The lordotic segment is distracted dorsally, and the diameter of the canal and foramen increases. In my opinion, it's not a suitable treatment for higher grade stenosis.
Taylor: It is fundamental to differentiate the devices that act as pure extensional blockers from the devices that are able to stabilize both extensional and anterior flexion as well. The characteristics of the Diam are unique. The implant is attached to each adjacent spinous process through use of 2 independent laces/tethers. The tethers enable the implant to be stretched during flexion. This feature provides a certain range of flexional and extensional stability in the sagittal plane without affecting the center of rotation. A multitude of biomechanical tests have been conducted to confirm this.
Zucherman: We know from numerous in vivo and in vitro studies that the X-Stop enlarges the central and lateral spinal canal. Biomechanical studies in cadavers have also shown unloading of the discs and facets. Motion studies show diminished extension range. As mentioned, adding a strap will limit flexion also.

Question \#4. What are the primary indications for implantation of an interspinous process spacer? Are there specific conditions where these spacers cannot be implanted?

Adelt: The primary indication for the Coflex is back pain coming up from facet arthrosis. Due to the grade of arthrosis, synovial cysts may be an indication. Important is the exclusion of instability as determined by X-rays in flexion and extension.
Unstable segments cannot be treated by interspinous devices. I don't want to go into details of general problems such as osteoporosis, but this also may be an exclusion.
Taylor: The Diam device addresses the painful consequences of the posterior transfer of load which occurs during the natural aging process. The overloading of the facets results in arthropathy and static disorders, which can result in foraminal stenosis and/or retrolisthesis.
When there is no more spinous process available after breakage or a laminectomy, an IPD device cannot be implanted unless a reconstructive procedure can be attempted. On the other hand, an unstable spondylolisthesis contraindicates the recourse to any IPD device. Lastly, a rotational instability cannot be managed with an IPD device as a standalone.
Zucherman: The primary indication for the X-Stop device is spinal stenosis that produces back and/or leg pain with standing and walking. Patients should have relief of most of their symptoms in sitting and be able to sit for an hour comfortably. Additionally, they need to have localized disease to 1 or 2 segments and less than first-degree spondylolisthesis. Lytic spondylolisthesis at the treated level or the level below will make the X-Stop ineffective and is a contraindication.
Osteopenia, osteoporosis, and history of pathologic fractures are relative contraindications, and the force applied during insertions in a situation of softer bone must be diminished. We frequently augment the spinous process with polymethylmethacrylate (PMMA) injection in cases where we believe this is an issue. Frequently, at the L5-S1 level, the $\mathrm{S} 1$ spinous process is not large enough to support the device, and it is therefore contraindicated.

\section{Question \#5. In the degenerative cascade, where do you think this technology is best suited when compared to nuclea replacement, pedicle screw based dynamic devices, artificial discs, and fusion?}

Adelt: To find the place in the degenerative cascade of treatment you must be aware of the fact that all the other implants in this cascade are dealing with degenerative disc disease (DDD). The targets of interspinous spacers are the joints at the dorsal part of the column and not the disc, which is located far away ventrally. The Coflex is the implant that is nearest to the center of rotation, but its influence is at the dorsal annulus, while not affecting the disc and facets at adjacent levels due to its dynamic characteristics. Due to the less-invasive procedure and dynamic aspect, I think the devices are best suited at the beginning of the degenerative cascade, soon after non-invasive therapies. 
Taylor: An IPD device, such as the Diam, does not burn any bridges for potential surgeries that the patient may require in the future. For this reason, an IPD device can be suggested at an earlier stage in the disease process in order to partially offload painful structures, such as the facets, as well as the posterior part of the disc. An IPD device is a low-risk surgical alternative for patients not responding properly to conservative treatment. Clearly, this technology does not compete with any radical or end-stage surgery such as total disc replacement (TDR) or fusion.
Zucherman: Since these interspinous devices have an extremely low complication rate with very fast recovery, I consider them the treatment of choice in situations where they have an expected good success rate. So far, it has not been demonstrated with evidence-based medicine that they are effective in discogenic pain and central back pain that is not caused by a spinal stenosis and a variant of neurogenic claudication. Since none of the interspinous process devices burn the bridges for the other procedures mentioned above, they can be more readily considered in appropriate situations.

Question \#6. What evidence of effectiveness is being recorded in current trials? What are the primary complications being observed and at what rates?

Adelt: Prospective randomized trials have started in the US and in Germany. To date we just have retrospective trials. In our own series of 240 patients, an improvement in low-back pain was shown in $72 \%$ with $40 \%$ demonstrating complete relieve. An improvement in walking distance due to back pain was found in $87 \%$ of the patients. There was a high rate of patient satisfaction with $94 \%$ being satisfied or very satisfied and $95 \%$ indicating they would have surgery again. One difficulty is unrecognized instabilities which lead to later fusion operations. With all interspinous devices, there are possible postoperative hematomas because of the "dead space." We always use a drain with a Coflex.
Taylor: The Diam has been implanted since 1997, and more than 45,000 cases have been reported. Retrospective studies were conducted, and the conclusions look encouraging, especially for stenosis, disc herniation (DH) in young patients displaying pre-existing low-back pain (LBP), and for a specific low back (LB) syndrome. Three randomized multicenter prospective studies are underway in order to confirm these applications.

The 3 US Food and Drug Administration investigational device exemption (IDE) studies underway with the Diam device are as follows:

IDE Study \#1. Diam \& Decompression vs. Decompression: A prospective, randomized, controlled pivotal clinical investigation of the Diam System in patients with degenerative lumbar spinal stenosis (DLSS). The study is designed as a superiority study over decompression alone, based on a primary endpoint of overall success based on Oswestry score and neurological success improvement. It is being studied in 30 sites, on 660 patients, and is a 1:1 randomization. The Diam device is being added to a decompression surgery in an effort to address the low-back pain component of DLSS.

IDE Study \#2. Diam vs. Conservative Care: A prospective, randomized, controlled pivotal clinical investigation of the Diam System in patients with moderate lumbar degenerative disc disease (LDDD). The study is designed as a superiority study over conservative care, based on a primary endpoint of overall success using Oswestry score improvement. It is being studied in 20 sites, on 306 patients, and is a 2:1 randomization. The Diam device is being studied to understand its impact in improving back pain due to LDDD vs that of nonoperative care.

IDE Study \#3 (Outside US). Diam \& Discectomy vs. Discectomy: A prospective, randomized, controlled pivotal clinical investigation of the Diam System in patients with DLSS. The study is designed as a non-inferiority study over discectomy alone, based on a primary endpoint of a demonstrated clinically meaningful difference in reduction of disability based on visual analog scale (VAS) back pain scores. It is being studied in 20 sites, on 268 patients, and is a 1:1 randomization. The Diam device is being added to a discectomy surgery in an effort to address the low-back pain component of DLSS.

Some intraoperative fractures of the spinous process occurred at the very beginning of our OUS (outside US) experience. A few cases of liquid collection were leading to a revision and were analyzed as allergic reaction. A rate of around $6 \%$ of patients underwent revision surgery for insufficiency of pain relief. No neurologic complications in relation to the Diam have been reported. 
Zucherman: Although I am a Wallis device (Abbott Spine, Austin, Texas) investigator, I have no experience with it to date, nor have I seen the data regarding complications in the US trials.

Our extensive experience with the X-Stop has shown that the complication rates are extremely low compared to any other spinal procedure. In the Food and Drug Administration investigational device exemption (IDE) study, the complication rate was the same as for epidural injection. In our own experience since the X-Stop has been released for general use, we have been following approximately
400 cases with prospective data collection and have found that about $50 \%$ of the patients are very satisfied and are essentially cured, and $20 \%$ are somewhat satisfied, with $30 \%$ of the patients being somewhat dissatisfied or unsatisfied. Comorbidities are extremely common, as the patient population's average age runs in the $70 \mathrm{~s}$. The most common complication is ineffectiveness of the device. Common causes for this are multilevel disease not fully treated, other degenerative problems aside from stenosis that persist, and extension of the indications beyond the labeled ones in which the device is less effective, such as larger degrees of spondylolisthesis and coronal-plane deformities.

\section{Question \#7. How long do you think these devices would last and what is your revision strategy?}

Adelt: In our study which originally spanned the years 2002 to 2005 (now up to 2008), we have not seen any broken implants, only one deformed implant. In the laboratory, there was no material fatigue noticed, which hints at the duration of the life of Coflex in the long term.
The revision strategy is easy; there is no problem with removing Coflex even after years. With a chisel you remove overgrowing bone, especially at the wings. When you open the wings with that chisel, you can pull out the implant with pliers.
Taylor: The indications have been refined, and the technology has evolved. Therefore, the follow-up is not homogenous. Our understanding concludes that these devices allow us to address the pathology sooner, thereby averting too extensive/ invasive a surgery.
At the moment, revision is relatively invasive when removing the IPD device and switching to pedicle screws or intersomatic procedures. We aim to have IPDs continue to develop and be able to address more advanced pathology in the future.
Zucherman: Our first group of patients, with procedures performed in 1997, showed no deterioration in the effectiveness of the devices over time. In another study published by Idler1 with approximately 6-year follow-up, the spinal canals after treatment with the X-Stop devices were still larger than preoperatively. My general impression is that if the devices are effective for the first 6 to 8 months, it is unusual for them to become ineffective. The general need for recurrent surgery for spinal stenosis is about 3\% per year; I believe this is around the percentage of X-Stop patients who require reoperating as well.
Our revision strategy depends on the postoperative course. For patients who have had an obviously beneficial effect and then lost the effect, we would suspect that settling had occurred, or there is a new abnormality at the same level or a different level. In these cases, we contemplate revision with larger X-Stop devices. In cases where relief is partial and residual symptoms persist, and there is multilevel disease, we could consider treating the untreated levels and revising the previously treated levels with larger devices. In cases in which all the levels are treated and the response is unsatisfactory, we usually recommend removal of the device and laminectomy with or without fusion.

\section{REFERENCES}

1. Idler C, Zucherman JF, Yerby S, Hsu KY, Hannibal M, Kondrashov D. A novel technique of intra-spinous process injection of PMMA to augment the strength of an inter-spinous process device such as the X Stop. Spine. 2008;33(4):452-456. 